\title{
Pharmacokinetics and safety of intravenously administered citrulline in children undergoing congenital heart surgery: Potential therapy for postoperative pulmonary hypertension
}

Frederick E. Barr, MD, MSci, ${ }^{a}$ Rommel G. Tirona, PhD, ${ }^{\mathrm{b}}$ Mary B. Taylor, MD, MSci, ${ }^{a}$ Geraldine Rice, RN, BSN, ${ }^{a}$ Judith Arnold, RN, BSN, ${ }^{a}$ Gary Cunningham, MS, ${ }^{a}$ Heidi A. B. Smith, MD, MSci, ${ }^{a}$ Adam Campbell, BS, ${ }^{a}$ Jeffrey A. Canter, MD, MPH, ${ }^{\mathrm{e}}$ Karla G. Christian, MD, ${ }^{\mathrm{c}}$ Davis C. Drinkwater, MD, ${ }^{\mathrm{c}}$ Frank Scholl, MD, ${ }^{\mathrm{c}}$ Ann Kavanaugh-McHugh, MD, ${ }^{d}$ and Marshall L. Summar, MD $^{\mathrm{e}}$

From the Departments of Pediatrics, Pediatric Critical Care, ${ }^{\mathrm{a}}$ Pharmacology, ${ }^{\mathrm{b}}$ Cardiothoracic Surgery, ${ }^{\mathrm{c}}$ and Pediatric Cardiology, ${ }^{\mathrm{d}}$ and the Center for Human Genetics Research, ${ }^{\text {e }}$ Vanderbilt Children's Hospital, Vanderbilt University Medical Center, Nashville, Tenn.

Supported by the National Institutes of Health, National Heart, Lung, and Blood Institute, grant RO1 HL073317 (F.E.B.).

Received for publication Oct 27, 2006; revisions received Jan 11, 2007; accepted for publication Feb 1, 2007.

Address for reprints: Frederick E. Barr, MD, MSci, 2200 Children's Way, 5121 Doctor's Office Tower, Nashville TN 37232-9075 (E-mail: rick.barr@ vanderbilt. edu).

J Thorac Cardiovasc Surg 2007;134:319-26 $0022-5223 / \$ 32.00$

Copyright () 2007 by The American Association for Thoracic Surgery

doi:10.1016/j.jtcvs.2007.02.043
Objective: Pulmonary hypertension may complicate surgical correction of congenital heart defects, resulting in increased morbidity and mortality. We have previously shown that plasma levels of the nitric oxide precursors citrulline and arginine drop precipitously after congenital cardiac surgery and that oral citrulline supplementation may be protective against the development of pulmonary hypertension. In this study, we assessed the safety and pharmacokinetic profile of intravenous citrulline as a potential therapy for postoperative pulmonary hypertension.

Methods: The initial phase of this investigation was a dose-escalation study of intravenously administered citrulline in infants and children undergoing one of five congenital cardiac surgical procedures (phase 1). The primary safety outcome was a 20\% drop in mean arterial blood pressure from the baseline pressure recorded after admission to the intensive care unit. Based on our previous work, the target circulating plasma citrulline trough was 80 to $100 \mu \mathrm{mol} / \mathrm{L}$. Each patient was given two separate doses of citrulline: the first in the operating room immediately after initiation of cardiopulmonary bypass and the second 4 hours later in the pediatric intensive care unit. Stepwise dose escalations included $50 \mathrm{mg} / \mathrm{kg}, 100 \mathrm{mg} / \mathrm{kg}$, and $150 \mathrm{mg} / \mathrm{kg}$. After model-dependent pharmacokinetic analysis, we enrolled an additional 9 patients (phase 2) in an optimized dosing protocol that replaced the postoperative dose with a continuous infusion of citrulline at $9 \mathrm{mg} /(\mathrm{kg} \cdot \mathrm{h})$ for 48 hours postoperatively.

Results: The initial stepwise escalation protocol (phase 1) revealed that an intravenous citrulline dose of $150 \mathrm{mg} / \mathrm{kg}$ given after initiation of cardiopulmonary bypass yielded a trough level of in the target range of approximately 80 to $100 \mu \mathrm{mol} / \mathrm{L} 4$ hours later. The postoperative dose revealed that the clearance of intravenously administered citrulline was $0.6 \mathrm{~L} /(\mathrm{h} \cdot \mathrm{kg})$, with a volume of distribution of $0.9 \mathrm{~L} / \mathrm{kg}$ and estimated half-life of 60 minutes. Because of the short half-life, we altered the protocol to replace the postoperative dose with a continuous infusion of $9 \mathrm{mg} /$ $(\mathrm{kg} \cdot \mathrm{h})$. An additional 9 patients were studied with this continuous infusion protocol (phase 2). Mean plasma citrulline levels were maintained at approximately 125 $\mu \mathrm{mol} / \mathrm{L}$, with a calculated clearance of $0.52 \mathrm{~L} /(\mathrm{h} \cdot \mathrm{kg})$. None of the 17 patients studied had a $20 \%$ drop in mean arterial blood pressure from baseline.

Conclusions: In this first report of the use of intravenous citrulline in humans, we found citrulline to be both safe and well tolerated in infants and young children undergoing congenital cardiac surgery. Because of the rapid clearance, the optimal dosing regimen was identified as an initial bolus of $150 \mathrm{mg} / \mathrm{kg}$ given at the initiation of cardiopulmonary bypass, followed 4 hours later by a postoperative infusion of $9 \mathrm{mg} /(\mathrm{kg} \cdot \mathrm{h})$ continued up to 48 hours. Using this regimen, plasma arginine, citrulline, and nitric oxide metabolite levels were well maintained. Intravenous citrulline needs to be studied further as a potential therapy for postoperative pulmonary hypertension. 
$\mathrm{P}$ ulmonary hypertension is a potential complication after surgical correction of congenital cardiac defects that has been associated with increased postoperative morbidity and mortality. ${ }^{1-5}$ Current perioperative treatment includes the use of inhaled nitric oxide and several nonselective pulmonary vasodilators, including milrinone, epoprostenol, sildenafil citrate (INN sildenafil), and generation of alkalosis. Another potential therapy includes increasing endogenous nitric oxide synthesis by supplementation with citrulline or arginine. ${ }^{6,7}$ Nitric oxide is produced from L-citrulline and L-arginine, amino acids generated through the urea cycle (Figure 1). ${ }^{8-10}$ We have previously demonstrated that citrulline and arginine levels drop precipitously after surgical correction of congenital cardiac defects with cardiopulmonary bypass and do not recover for as long as 48 hours after surgery. ${ }^{11}$ In addition, children with postoperative pulmonary hypertension had significantly lower plasma arginine levels than those without this complication. We subsequently conducted a randomized, placebocontrolled trial of oral citrulline supplementation in this same patient population and found that although absorption was variable, citrulline was safe and that children who had a plasma citrulline level greater than $40 \mu \mathrm{mol} / \mathrm{L}$ at 12 hours after surgery were free from postoperative pulmonary hypertension. ${ }^{10}$

Intravenously administered citrulline has not been previously studied in human beings. The hypothesis of this study was that intravenously administered citrulline would be safe and would prevent the postoperative drop in plasma citrulline and arginine levels noted in our previous observational studies.

\section{Materials and Methods Patient Enrollment}

Approval from Vanderbilt's institutional review board (IRB) was obtained before patient enrollment. A total of 17 patients were enrolled in this open-label dose-escalation study at Vanderbilt Children's Hospital between May 2005 and January 2006.

All infants or children younger than 6 years undergoing one of five surgical procedures for correction of congenital heart lesions were considered for enrollment. The eligible surgical procedures were as follows: (1) repair of atrioventricular septal defect, (2) repair of ventricular septal defect, (3) bidirectional Glenn procedure (superior cavopulmonary shunt), (4) modified Fontan procedure (total cavopulmonary connection), and (5) arterial switch procedure. Exclusion criteria were as follows: (1) significant pulmonary arterial narrowing not addressed surgically, (2) previous pulmonary artery stent placement, (3) previous pulmonary artery angioplasty, (4) significant left-sided atrioventricular valve regurgitation, (5) pulmonary venous return abnormalities, (6) pulmonary vein stenosis, (7) preoperative mechanical ventilation, and (8) preoperative inotropic infusions.

Informed written consent was obtained from parents of the enrolled patients during preoperative evaluation at the Cardiotho-
Abbreviations and Acronyms
DSMB $=$ data safety monitoring board
FDA $=$ Food and Drug Administration
IRB = institutional review board
$\mathrm{k}_{\mathrm{rem}}=$ constant of citrulline removal
PICU = pediatric intensive care unit
$\mathrm{R}_{\mathrm{app}}=$ rate of citrulline appearance

racic Surgery Clinic (outpatient) or at Vanderbilt Children's Hospital (inpatient). Three cardiac surgeons at Vanderbilt Children's Hospital (K.G.C., D.C.D., F.S.) performed the surgical procedures with similar cardiopulmonary bypass and cardioplegia preparations.

This study was monitored closely by a data safety monitoring board (DSMB) composed of a pediatric cardiologist, a pediatric critical care physician, and a general pediatrician. The DSMB met three times during the study to review the safety and pharmacokinetic data.

\section{Adverse Events}

Intravenous citrulline administration carries a theoretic risk of systemic arterial hypotension. An adverse drop in mean arterial pressure was defined as a decrease of more than $20 \%$ from baseline. The baseline postoperative mean arterial blood pressure was calculated as the average of mean arterial blood pressure measurements collected every 5 minutes for the 30 minutes immediately before the administration of the postoperative dose or infusion. The bedside monitor was then set to alarm if that 20\% drop was reached at any time in the 48-hour study period. If the bedside monitor reached the preset limit and alarmed, the beside nurse was instructed to alert the study physician or nurse and to record mean arterial pressure every 5 minutes for 30 minutes. If the average of these 5-minute recordings was $20 \%$ below the original baseline blood pressure, the citrulline was discontinued. Patients were treated for hypotension at the discretion of the clinical pediatric intensive care unit (PICU) staff with volume resuscitation, inotropic or vasopressor support, or both. Development of hypotension according to these criteria that necessitated discontinuation of citrulline was counted as an adverse event and was reported to the DSMB, IRB, and Food and Drug Administration (FDA).

Serious adverse events, such as cardiac arrest, need for extracorporeal membrane oxygenation, and death, were reported immediately to the DSMB, the Vanderbilt IRB, and the FDA.

\section{Study Protocol}

The study design for the first 8 patients (phase 1) was a doseescalation protocol with two intravenously administered bolus doses of citrulline to determine the optimal dose and characterize pharmacokinetic parameters including half-life, clearance, and volume of distribution. The first bolus (given during the course of 10 minutes) was administered after initiation of cardiopulmonary bypass in the operating room; and the second (given during the course of $30 \mathrm{~min}$ ) was administered 4 hours later in the critical care unit. The intravenous doses of citrulline for each bolus were 50 $\mathrm{mg} / \mathrm{kg}$ (2 patients), $100 \mathrm{mg} / \mathrm{kg}$ ( 2 patients), and $150 \mathrm{mg} / \mathrm{kg}$ (4 patients). 


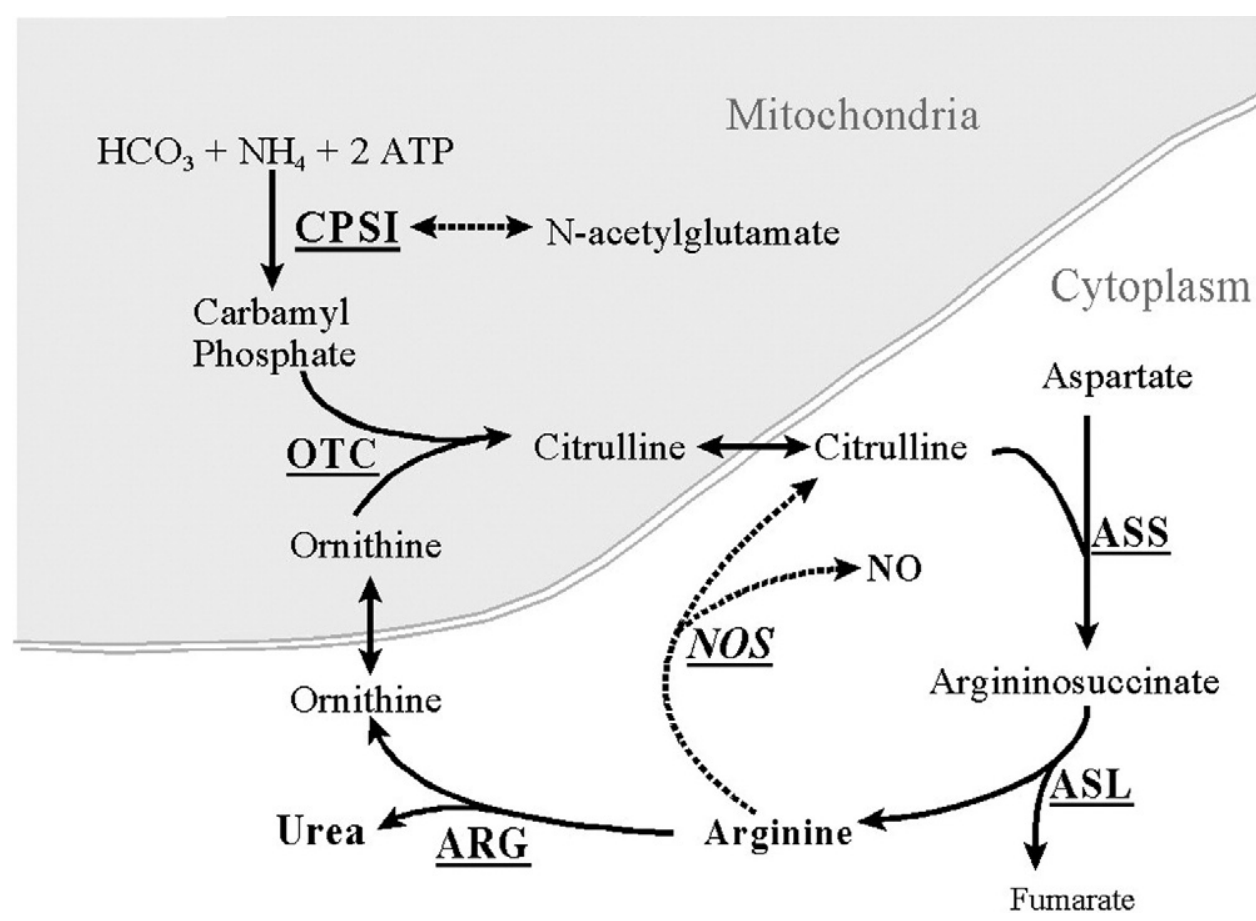

Figure 1. Carbamyl phosphate synthetase I (CPSI) is rate-limiting enzyme of urea cycle. Polymorphisms in its gene alter availability of nitric oxide precursors, citrulline, and arginine. Citrulline is metabolized into arginine, which

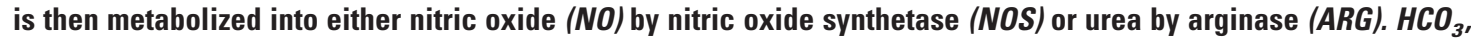
Bicarbonate; $\mathrm{NH}_{4}$, ammonium; ATP, adenosine triphosphate; OTC, ornithine transcarbamylase; ASS, argininosuccinate synthetase; $\boldsymbol{A S L}$, argininosuccinate lyase. From Smith HA, Canter JA, Christian KG, Drinkwater DC, Scholl FG, Christman BW, et al. Nitric oxide precursors and congenital heart surgery: a randomized controlled trial of oral citrulline. J Thorac Cardiovasc Surg. 2006;132:58-65.

The study design was changed (phase 2) after it was determined that the half-life and clearance were not compatible with intermittent dosing. After consultation with a pharmacologist, the next 9 patients received a $150-\mathrm{mg} / \mathrm{kg}$ intravenously administered bolus of citrulline during the course of $10 \mathrm{~min}$ in the operating room after initiation of cardiopulmonary bypass, followed 4 hours later in the critical care unit by a continuous infusion of $9 \mathrm{mg} /(\mathrm{kg} \cdot \mathrm{h})$ that continued for 48 hours.

The citrulline preparation was provided by the Investigational Drug Service of the Vanderbilt Hospital Clinical Pharmacy. Citrulline was administered as a $50-\mathrm{mg} / \mathrm{mL}(5 \%)$ isotonic solution, with distilled water as a suspending agent.

\section{Sample Collection}

A 3-mL sample of blood was obtained from each patient at selected time points. In phase 1 , samples were collected immediately after initiation of cardiopulmonary bypass before the first bolus given in the operating room, postoperatively 4 hours after the operating room bolus (immediately before the postoperative bolus), and then 1, 2, 3, 4, and 12 hours after the postoperative bolus. In phase 2, samples were collected immediately after initiation of cardiopulmonary bypass (before the first bolus given in the operating room), immediately after the operating room bolus, postoperatively 4 hours after the operating room bolus (immediately before the postoperative infusion), and then $6,12,24$, and 48 hours after initiation of the postoperative continuous infusion. Samples were collected in citrated tubes, placed on ice, and stored at $4{ }^{\circ} \mathrm{C}$ until processing. Samples were centrifuged for separation of plasma and cellular components. Plasma samples were frozen at $-70^{\circ} \mathrm{C}$ until further laboratory analysis.

\section{Laboratory Measurements}

Concentrations of plasma citrulline and arginine were determined through amino acid analysis by cation-exchange chromatography with a Beckman 7300 amino acid analyzer (Beckman Coulter, Inc, Fullerton, Calif). Calibration of the analyzer with known standards was completed before testing of patient samples.

Nitric oxide metabolites were measured by chemiluminescence with a Sievers 280 nitric oxide analyzer (GE Analytical Instruments, Boulder, Colo). Plasma samples were mixed 1:2 sample/ cold ethanol at $0^{\circ} \mathrm{C}$ for 30 minutes. After centrifugation at 14,000 rpm for 5 minutes, samples were injected into the analyzer. This method relies on catalytic reduction of nitric oxide metabolites by exposure to warm vanadium hydrochloride. Liberated nitric oxide was driven by nitrogen gas into an ozone chamber. Light released by nitric oxide-ozone interaction was captured by a photomultiplier tube and relayed to an analytic software program. A standard curve with sodium nitrite was used to determine sample concentrations. 


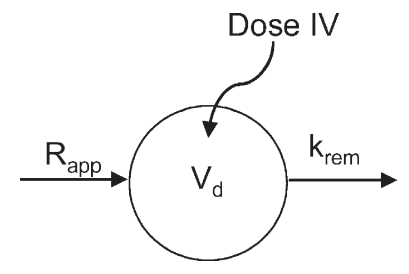

Figure 2. Pharmacokinetic model used in analysis of phase 1 data and for design of phase 2 intravenous (IV) dosing regimen. In this model, body is represented as single compartment with volume of distribution $\left(\mathbf{V}_{\mathrm{d}}\right)$. Citrulline appearance in plasma is described by zero-order process $\left(\boldsymbol{R}_{\text {app }}\right)$ to account for endogenous production, whereas removal from plasma is determined by firstorder process $\left(\boldsymbol{k}_{\text {rem }}\right)$.

\section{Pharmacokinetic Analysis}

Data obtained for each patient in phase 1 were fitted to the pharmacokinetic model depicted in Figure 2. In this model, the body exists as a single compartment with a volume of distribution. The appearance of citrulline in plasma is described by a zero-order process (rate of citrulline appearance, $\mathrm{R}_{\mathrm{app}}$ ) to account for endogenous production, whereas the removal of citrulline is determined by a first-order process (constant of citrulline removal, $\mathrm{k}_{\mathrm{rem}}$ ). It is assumed that the values of all parameters remained constant for each patient during the course of plasma sampling. Mass-balance differential equations were inputted into SCIENTIST (MicroMath Scientific Software, St Louis, Mo) and solved numerically. Data fitting was accomplished by a weighted, least squares procedure to obtain the simultaneous estimates of $\mathrm{R}_{\mathrm{app}}, \mathrm{k}_{\mathrm{rem}}$, and volume of distribution. Clearance was calculated from $\mathrm{k}_{\mathrm{rem}}$ multiplied by the volume of distribution, and half-life was estimated as $\ln 2 / \mathrm{k}_{\mathrm{rem}}$.

\section{Results}

\section{Patient Enrollment}

Seventeen patients were successfully enrolled, 8 patients in phase 1 and 9 in phase 2 . The median age of the patients was 6 months (interquartile range 3.6-30.6 months), with $55 \%$ male and $81 \%$ white. Surgical interventions were as follows: 4 patients with ventricular septal defect repair, 8 patients with atrioventricular septal defect repair, 2 patients with bidirectional Glenn shunt, 1 patient with modified Fontan procedure, and 2 patients with arterial switch.

\section{Safety}

There were no significant adverse events in phase 1 . There was 1 significant adverse event in phase 2 . That patient underwent an atrioventricular septal defect repair and was in a junctional rhythm postoperatively, necessitating atrial pacing. At approximately 8 postoperative hours, the patient showed the acute onset of profound bradycardia consistent with complete heart block that was not preceded by systemic hypotension and was not responsive to ventricular pacing. Advanced life-support measures were instituted, including open cardiac massage and emergency cannulation for venoarterial extracorporeal membrane oxygenation, which was required for 48 hours. The patient subsequently recovered fully and was discharged home on hospital day 22. The DSMB reviewed the case and determined that the significant adverse event was unlikely to be related to the citrulline administration. The adverse event was also reported to the Vanderbilt IRB, the National Institutes of Health, and the FDA.

\section{Pharmacokinetics}

Phase 1. Patients in phase 1 were given two doses of intravenously administered citrulline, the first just after initiation of cardiopulmonary bypass and the second 4 hours later after admission to the PICU. The plasma levels of citrulline at the various times after drug administration are depicted in Figure 3. Patients 1 and 2 received $50-\mathrm{mg} / \mathrm{kg}$ citrulline intravenously and had a peak citrulline level of approximately $220 \mu \mathrm{mol} / \mathrm{L}$ and a 4-hour trough level of 40 $\mu \mathrm{mol} / \mathrm{L}$. No adverse side effects were noted. This trough was well below the target range of 80 to $100 \mu \mathrm{mol} / \mathrm{L}$, and the dose was subsequently increased. Patients 3 and 4 received $100-\mathrm{mg} / \mathrm{kg}$ citrulline intravenously and had a peak citrulline level of $375 \mu \mathrm{mol} / \mathrm{L}$ and a 4-hour trough of 50 $\mu \mathrm{mol} / \mathrm{L}$. Again, no adverse side effects were noted. This trough was also below the target range of 80 to $100 \mu \mathrm{mol} / \mathrm{L}$, and the dose was subsequently increased. Patient 5, 6, 7, and 8 received $150 \mathrm{mg} / \mathrm{kg}$ citrulline intravenously and had a peak citrulline level of $660 \mu \mathrm{mol} / \mathrm{L}$ and a 4-hour trough of $80 \mu \mathrm{mol} / \mathrm{L}$. This 4 -hour trough was in the target range of 80 to $100 \mu \mathrm{mol} / \mathrm{L}$, and the dose was not escalated further. The citrulline pharmacokinetic parameter estimates for each of the three dosage levels are summarized in Table 1, and the pharmacokinetic profiles are displayed in Figure 3. The half-life was calculated to be approximately 60 minutes, which was too short to proceed with intermittent dosing. The study design was changed to a bolus dose followed by a continuous infusion in phase 2 .

Phase 2. An additional 9 patients were enrolled in phase 2. On the basis of parameter estimates obtained from phase 1 (Table 1), pharmacokinetic simulations predicted that a bolus dose of $150 \mathrm{mg} / \mathrm{kg}$ followed 4 hours later by a continuous infusion of $9 \mathrm{mg} /(\mathrm{kg} \cdot \mathrm{h})$ would yield sustained plasma citrulline levels of approximately 80 to $100 \mu \mathrm{mol} / \mathrm{L}$ (Figure 4). Initiation of the continuous infusion was deliberately set at 4 hours after the bolus to allow sustained increased levels during separation from cardiopulmonary bypass and ultrafiltration, to allow time for admission to the PICU, and to allow postoperative hemodynamic stabilization to allow accurate assessment of the drug safety profile. The phase 2 mean plasma citrulline profile is shown in Figure 5. For the entire group, postoperative mean plasma citrulline levels were sustained at approximately 150 to 250 


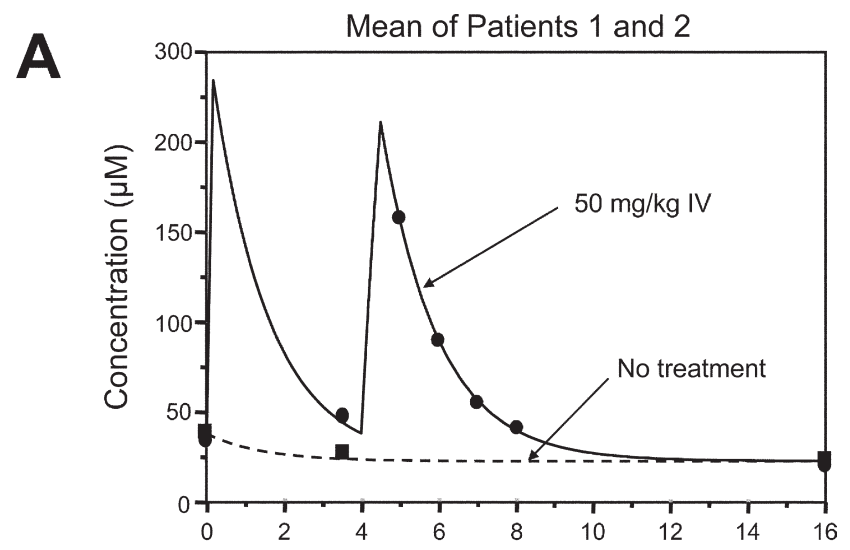

B
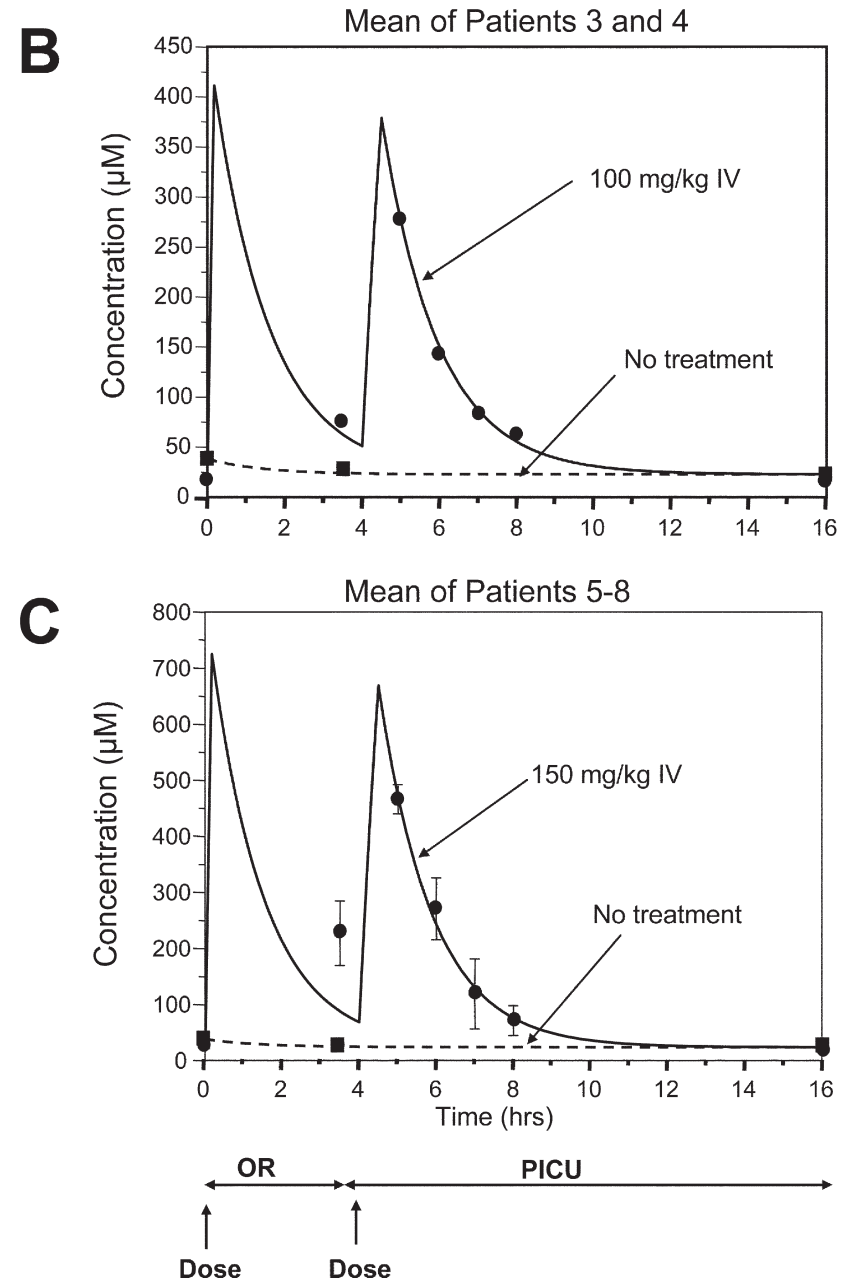

Figure 3. Pharmacokinetic profile of intravenously administered (IV) bolus-dose citrulline in phase 1. Dose-escalation study design in which each patient was given two boluses of citrulline, first in operating room (OR) on cardiopulmonary bypass and second 4 hours later after admission to pediatric intensive care unit (PICU). Patients 1 and 2 received $50 \mathrm{mg} / \mathrm{kg}$ (A), patients 3 and 4 received $100 \mathrm{mg} / \mathrm{kg}(B)$, and patients 5, 6, 7, and 8 received 150 $\mathrm{mg} / \mathrm{kg}$ (C). No adverse side effects were noted. $\mu \mathrm{mol} / \mathrm{L}$ during the 48-hour study period. Estimated citrulline clearance was $0.52 \pm 0.28 \mathrm{~L} /(\mathrm{kg} \cdot \mathrm{h})$ in phase 2 . The mean plasma citrulline levels of a previously published cohort of patients undergoing congenital cardiac surgery who had not received intravenously administered citrulline are also depicted in Figure 5 for comparison. ${ }^{11}$ Figure 6 depicts similar data for plasma arginine levels, which were also sustained after intravenous administration of citrulline relative to data from the previous cohort. ${ }^{11}$ Figure 7 depicts similar data for plasma nitric oxide metabolite levels, which were also sustained after intravenous administration of citrulline relative to data from the previous cohort. ${ }^{11}$

\section{Discussion}

This is the first report of the use of intravenously administered citrulline in human beings. The goal of this study was to determine the safety profile and pharmacokinetics of intravenously administered citrulline in a fairly unique patient population of infants and children undergoing surgical correction of congenital heart defects. This was an openlabel study that was not placebo controlled and thus not designed to evaluate the use of intravenously administered citrulline as a potential therapy for postoperative pulmonary hypertension. The pharmacokinetic and safety information gained from this study, however, has been subsequently used to design a randomized, placebo-controlled efficacy trial of intravenously administered citrulline for the treatment of postoperative pulmonary hypertension.

With regard to safety, we were primarily interested in systemic hypotension as a potential negative side effect of citrulline treatment. We defined systemic hypotension as a sustained $20 \%$ drop in mean arterial pressure from an average baseline recording that was obtained during the 30 minutes just before initiation of intravenous citrulline administration, either as a bolus (phase 1) or as a continuous infusion (phase 2). This definition took into account some of the inherent blood pressure variation in children immediately after congenital cardiac surgery. None of the patients in phase 1 met this definition of systemic hypotension. Only 1 patient in phase 2 of this study met this definition, and that patient's hypotension was precipitated by profound bradycardia and heart block, a side effect unlikely to be precipitated by citrulline. The DSMB reviewed that patient's clinical data and determined that the adverse event was unlikely to be related to the citrulline infusion. The Vanderbilt IRB, the National Institutes of Health, and the FDA were also notified and concurred with this conclusion.

The pharmacokinetics of intravenously administered citrulline in this patient population are significantly complicated by the interposition of cardiopulmonary bypass and ultrafiltration. We initially decided to administer a fairly large bolus of citrulline immediately after initiation of cardiopulmonary bypass, with the goal of providing a 4-hour 
TABLE 1. Model-dependent citrulline pharmacokinetic parameter estimates

\begin{tabular}{lccc}
\hline & Patients 1 and 2 & Patients 3 and 4 & Patients 5-8 \\
\hline Dose $(\mathrm{mg} / \mathrm{kg})$ & 50 & 100 & 150 \\
$\mathrm{R}_{\text {app }}(\mu \mathrm{mol} /[\mathrm{h} \cdot \mathrm{kg}])$ & $19.1(15.9,22.2)$ & $14.7(13.4,16.0)$ & $10.8 \pm 1.9$ \\
$\mathrm{k}_{\text {rem }}\left(\mathrm{h}^{-1}\right)$ & $0.78(0.67,0.89)$ & $0.72(0.87,0.56)$ & $0.68 \pm 0.16$ \\
Volume of distribution $(\mathrm{L} / \mathrm{kg})$ & $0.99(0.97,1.02)$ & $0.99(0.77,1.22)$ & $0.89 \pm 0.24$ \\
Clearance $(\mathrm{L} /[\mathrm{h} \cdot \mathrm{kg}])$ & $0.78(0.65,0.91)$ & $0.68(0.67,0.8)$ & $0.58 \pm 0.05$ \\
\hline
\end{tabular}

Data represent means of individual values $( \pm$ SD for patients 5 through 8; individual values are given in parentheses for patients 1 and 2 and patients 3 and 4) of parameter estimates from pharmacokinetic model shown in Figure 2. $R_{a p p}$, Rate of citrulline appearance; $k_{r e m}$, constant of citrulline removal.

target trough level of approximately 80 to $100 \mu \mathrm{mol} / \mathrm{L}$. This target level was intentionally above the threshold value of approximately $40 \mu \mathrm{mol} / \mathrm{L}$ that we had previously identified as potentially protective against postoperative pulmonary hypertension in our studies with orally administered citrulline. ${ }^{10}$ With an intravenously administered citrulline bolus of $150 \mathrm{mg} / \mathrm{kg}$, we were able to achieve that target 4-hour trough level. An alternative approach would have been to use a smaller initial bolus followed by an immediate continuous infusion, but the mechanics of cardiopulmonary bypass and ultrafiltration both during and after bypass made this approach impractical.

In phase 1 of the study, we determined that the halflife of intravenously administered citrulline is fairly short at approximately 60 minutes. The volume of distribution of citrulline was estimated between 0.8 and $1.0 \mathrm{~L} / \mathrm{kg}$ among the dosage groups in Phase 1 (Table 1). This value suggests that citrulline distributes to extravascular spaces. The high postdosing levels observed during phase 2 suggest that citrulline rapidly distributes out of the vascular space. The short half-life of citrulline is largely due to efficient elimination by the body, with clearance 0.6 to $0.8 \mathrm{~L} /(\mathrm{kg} \cdot \mathrm{h})$.

We did not study intravenously administered citrulline in patients who were not undergoing cardiac surgery. With computer modeling of data obtained from the second postoperative bolus dose in phase 1 of this study, however, we estimated that a bolus of $20 \mathrm{mg} / \mathrm{kg}$ immediately followed by a continuous infusion of $9 \mathrm{mg} /(\mathrm{kg} \cdot \mathrm{h})$ would rapidly achieve steady state at plasma levels of 100 $\mu \mathrm{mol} / \mathrm{L}$ in other patient groups at risk for pulmonary hypertension, in whom the effects of cardiopulmonary bypass would not be a concern in establishing the pharmacokinetics of citrulline. This noncardiac surgical protocol would need to be validated in further studies.

Determination of whether intravenously administered citrulline is a potential therapy for postoperative pulmonary hypertension will require a randomized clinical trial. We have recently initiated a trial of intravenously administered citrulline versus saline placebo according the described pro-

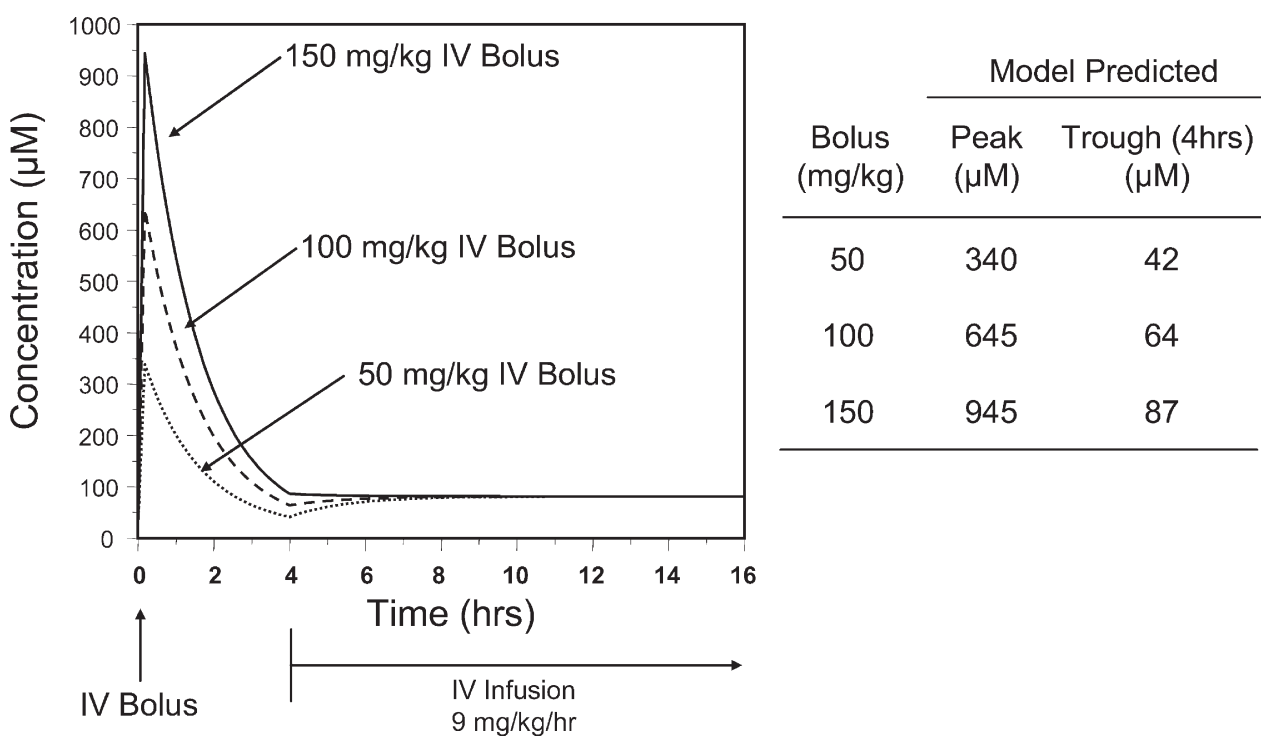

Figure 4. Pharmacokinetic modeling of intravenously administered (IV) bolus of citrulline given at beginning of surgery, followed 4 hours later by continuous infusion. Bolus dose of $150 \mathrm{mg} / \mathrm{kg}$ was determined most likely to yield 4-hour trough of 80 to $100 \mu \mathrm{mol} / \mathrm{L}$, and infusion of $9 \mathrm{mg} /(\mathrm{kg} \cdot \mathrm{h})$ was predicted to achieve steady state. This protocol was used in phase 2. 


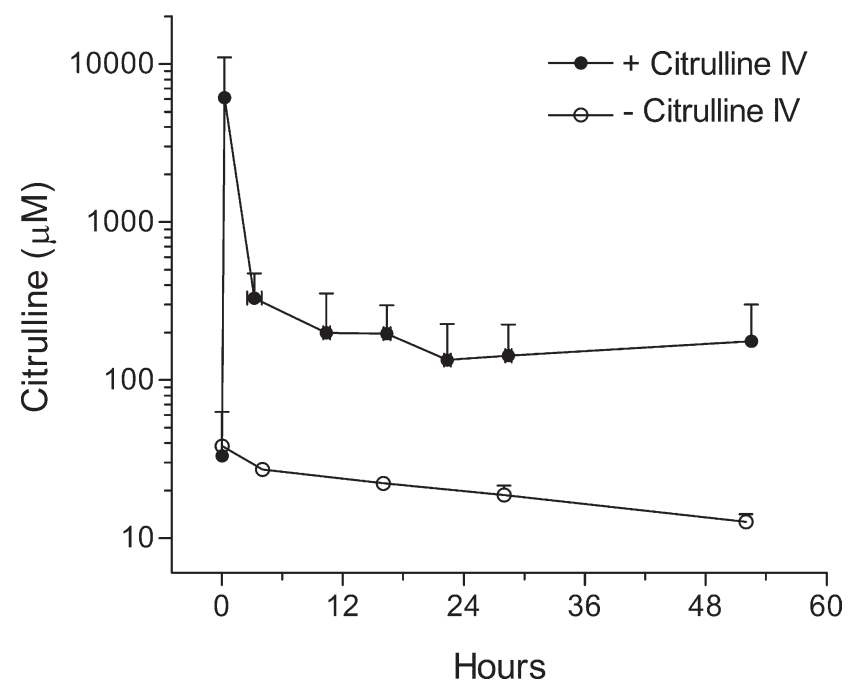

Figure 5. Plasma concentration-time profile of citrulline in phase 2. Patients 9 to 17 were administered citrulline $150 \mathrm{mg} / \mathrm{kg}$ intravenously (IV) in operating room on cardiopulmonary bypass, followed by continuous infusion at $9 \mathrm{mg} /(\mathrm{kg} \cdot \mathrm{h})$ initiated 4 hours later after admission to pediatric intensive care unit. Target citrulline levels were achieved with this citrulline dosing regimen. For comparison, citrulline plasma levels in infants undergoing similar procedures without citrulline treatment in previously published study are shown. ${ }^{11}$

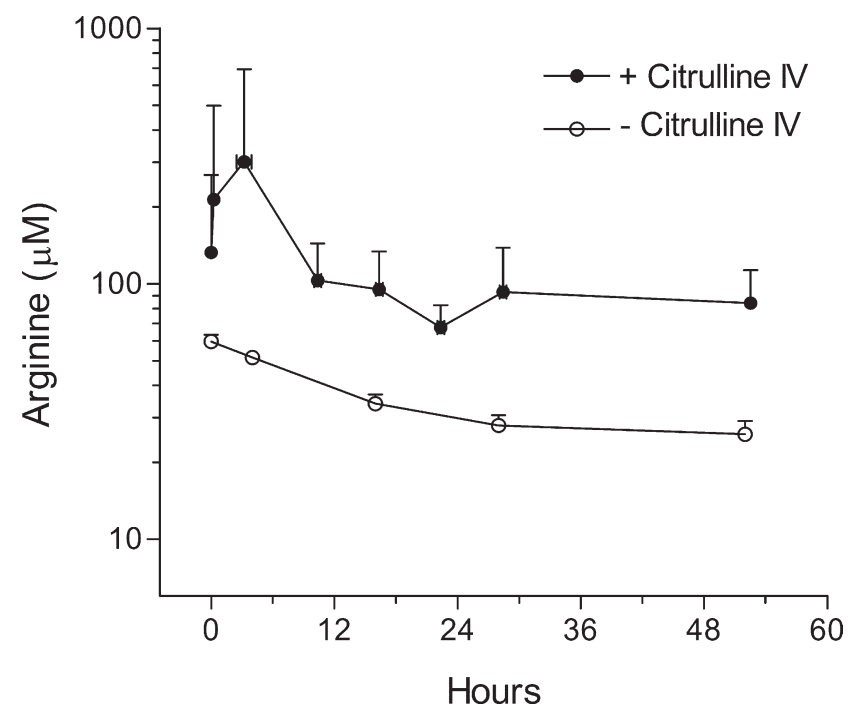

Figure 6. Plasma concentration-time profile of arginine in phase 2. Patients 9 to 17 were administered citrulline $150 \mathrm{mg} / \mathrm{kg}$ intravenously (IV) in operating room on cardiopulmonary bypass, followed by continuous infusion at $9 \mathrm{mg} /(\mathrm{kg} \cdot \mathrm{h})$ initiated 4 hours later after admission to pediatric intensive care unit. For comparison, arginine plasma levels in infants undergoing similar procedures without citrulline treatment in previously published study are shown. ${ }^{11}$

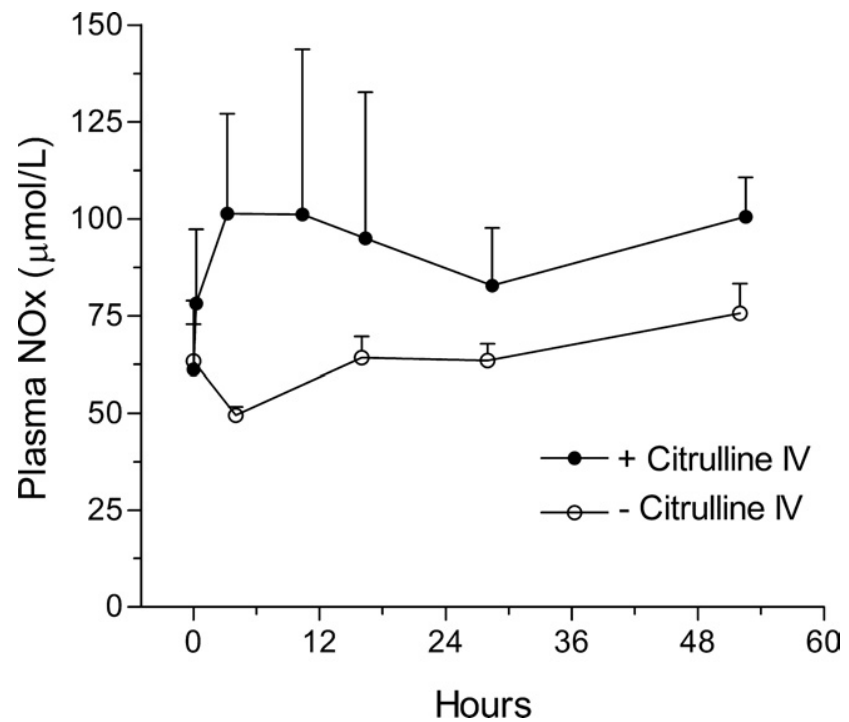

Figure 7. Plasma concentration-time profile of nitric oxide metabolites $(\mathrm{NOx})$ in phase 2. Patients $\mathbf{9}$ to $\mathbf{1 7}$ were administered citrulline $150 \mathrm{mg} / \mathrm{kg}$ intravenously (IV) in operating room on cardiopulmonary bypass, followed by continuous infusion at $9 \mathrm{mg} /(\mathbf{k g} \cdot \mathrm{h})$ initiated 4 hours later after admission to pediatric intensive care unit. For comparison, nitric oxide metabolite plasma levels in infants undergoing similar procedures without citrulline treatment in previously published study are shown. ${ }^{10}$

tocol in infants and children undergoing these same five cardiac surgical procedures, with a goal of reducing the incidence of postoperative pulmonary hypertension by $50 \%$. In addition, the efficacy of combination therapy of orally or intravenously administered citrulline and other therapies for pulmonary hypertension, such as inhaled nitric oxide, nebulized inhaled epoprostenol, and orally administered sildenafil citrate, should be investigated.

\section{References}

1. Steinhorn RH, Fineman JR. The pathophysiology of pulmonary hypertension in congenital heart disease. Artif Organs. 1999;23:970-4.

2. Bandla HP, Hopkins RL, Beckerman RC, Gozal D. Pulmonary risk factors compromising postoperative recovery after surgical repair for congenital heart disease. Chest. 1999;116:740-7.

3. Gentles TL, Mayer JE Jr, Gauvreau K, Newburger JW, Lock JE, Kupferschmid JP, et al. Fontan operation in five hundred consecutive patients: factors influencing early and late outcome. J Cardiovasc Surg. 1997;114:376-91.

4. Nakajima Y, Momma K, Seguchi M, Nakazawa M, Imai Y. Pulmonary hypertension in patients with complete transposition of the great arteries: midterm results after surgery. Pediatr Cardiol. 1996;17: 104-7.

5. Schulze-Neick I, Li J, Panny DJ, Reington AN. Pulmonary vascular resistance after cardiopulmonary bypass in infants: effect on postoperative recovery. J Thorac Cardiovasc Surg. 2001;121:1033-9.

6. Russell IA, Zwass M, Fineman JR, Balea M, Rouine-Rapp K, Brook $\mathrm{M}$, et al. The effects of inhaled nitric oxide on postoperative pulmo- 
nary hypertension in infants and children undergoing surgical repair of congenital heart disease. Anesth Analg. 1998;87:46-51.

7. Zobel G, Gamillscheg A, Schwinger W, Berger J, Urlesberger B, Dacar D, et al. Inhaled nitric oxide in infants and children after open heart surgery. J Cardiovasc Surg. 1998;39:79-86.

8. Moncada S, Higgs A. The L-arginine-nitric oxide pathway. $N$ Engl J Med. 1993;329:2002-12.

9. Pearson DL, Dawling S, Walsh WF, Haines JL, Christman BW, Bazyk A, et al. Neonatal pulmonary hypertension-urea-cycle intermediates, nitric oxide production, and carbamoyl-phosphate synthetase function. N Engl J Med. 2001;344:1832-8.

10. Barr FE, Beverley H, VanHook K, Cermak E, Christian K, Drinkwater D, et al. Effect of cardiopulmonary bypass on urea cycle intermediates and nitric oxide levels after congenital heart surge. J Pediatr. 2003;142:26-30.

11. Smith HA, Canter JA, Christian KG, Drinkwater DC, Scholl FG, Christman BW, et al. Nitric oxide precursors and congenital heart surgery: a randomized controlled trial of oral citrulline. $J$ Thorac Cardiovasc Surg. 2006;132:58-65. 